\title{
MIMO-less Space Division Multiplexing Transmission over 1 km Elliptical Core Few Mode Fiber
}

\author{
F. Parmigiani ${ }^{1}$, Y. Jung ${ }^{1}$, L. Grüner-Nielsen ${ }^{2}$, T. Geisler ${ }^{2}$, P. Petropoulos ${ }^{1}$, D. J. Richardson ${ }^{1}$ \\ ${ }^{1}$ Optoelectronics Research Centre, University of Southampton, SO17 1BJ, United Kingdom \\ ${ }^{2}$ OFS, Priorparken 680, 2605 Brøndby, Denmark \\ frp@orc.soton.ac.uk
}

\begin{abstract}
We experimentally demonstrate 10-Gbit/s OOK MIMO-less SDM transmission over $1 \mathrm{~km}$ of a three-spatial-mode elliptical-core fiber at $1550 \mathrm{~nm}$. Negligible power penalty is achieved thanks to the low modal crosstalk $(<-22 \mathrm{~dB})$ between any pair of the $\mathrm{LP}_{01}, \mathrm{LP}_{11 \mathrm{a}}$ and $\mathrm{LP}_{11 \mathrm{~b}}$ modes.

OCIS codes: (060.2330) Fiber optics communications; (060.4230) Multiplexing.
\end{abstract}

\section{Introduction}

Space division multiplexing (SDM) has been combined with wavelength division multiplexing to achieve a substantial increase in the transmission capacity of optical fibers, by transmitting independent information channels over different spatial cores or modes of a multi-core or few-mode fiber (FMF) [1]. Mode coupling in FMFs necessitates the use of complex and expensive multiple input multiple output (MIMO) digital signal processing (DSP) to allow successful reception of the signals over long transmission distances. When considering high capacity, short-reach data-center applications however, where both cost and power consumption need to be kept as low as possible, the use of MIMO-less direct detection is imperative, and this imposes stringent requirements on the tolerable cross-talk levels among the transmitted modes. Recently, the use of elliptical (multi-) core FMF has been proposed as a means to guarantee negligible mode coupling even between modes of the $\mathrm{LP}_{11}$ mode group that are normally degenerate [2-5]. Notably, MIMO-less SDM transmission using three-spatial-modes $\left(\mathrm{LP}_{01}, \mathrm{LP}_{11 \mathrm{a}}\right.$ and $\left.\mathrm{LP}_{1 \mathrm{~b}}\right)$ at $1.3 \mu \mathrm{m}$ and two-spatial- modes $\left(\mathrm{LP}_{01}\right.$ and $\left.\mathrm{LP}_{11 \mathrm{a}}\right)$ at $1.5 \mu \mathrm{m}$ was demonstrated in a (sub) km-long FMF with a core-ellipticity of about $40 \%$ [2]. The same authors also demonstrated $1.2 \mathrm{Tbit} / \mathrm{s}$ wavelength division multiplexing $(\mathrm{WDM})+\mathrm{SDM}$ transmission at $1.5 \mu \mathrm{m}$ using a four-core FMF with each core having an ellipticity of about $11 \%$ and supporting two spatial mode groups. However, these impressive results come with an increased power penalty when simultaneously exciting the $\mathrm{LP}_{01}$ and $\mathrm{LP}_{11 \mathrm{a}}$ modes.

In this work, we demonstrate MIMO-less SDM transmission at $1.55 \mu \mathrm{m}$ over $1 \mathrm{~km}$ of an FMF with a core ellipticity of $10 \%$ and supporting the three-spatial-modes, $\mathrm{LP}_{01}, \mathrm{LP}_{11 \mathrm{a}}$ and $\mathrm{LP}_{11 \mathrm{~b}}$. A negligible power penalty and no error floors was observed even when transmitting $10 \mathrm{Gbit} / \mathrm{s}$ on- off keying (OOK) signals in the two spatial modes of the same $\mathrm{LP}_{11}$ mode group, highlighting the overall low level of modal crosstalk.

\section{Experimental Set-up and Results}

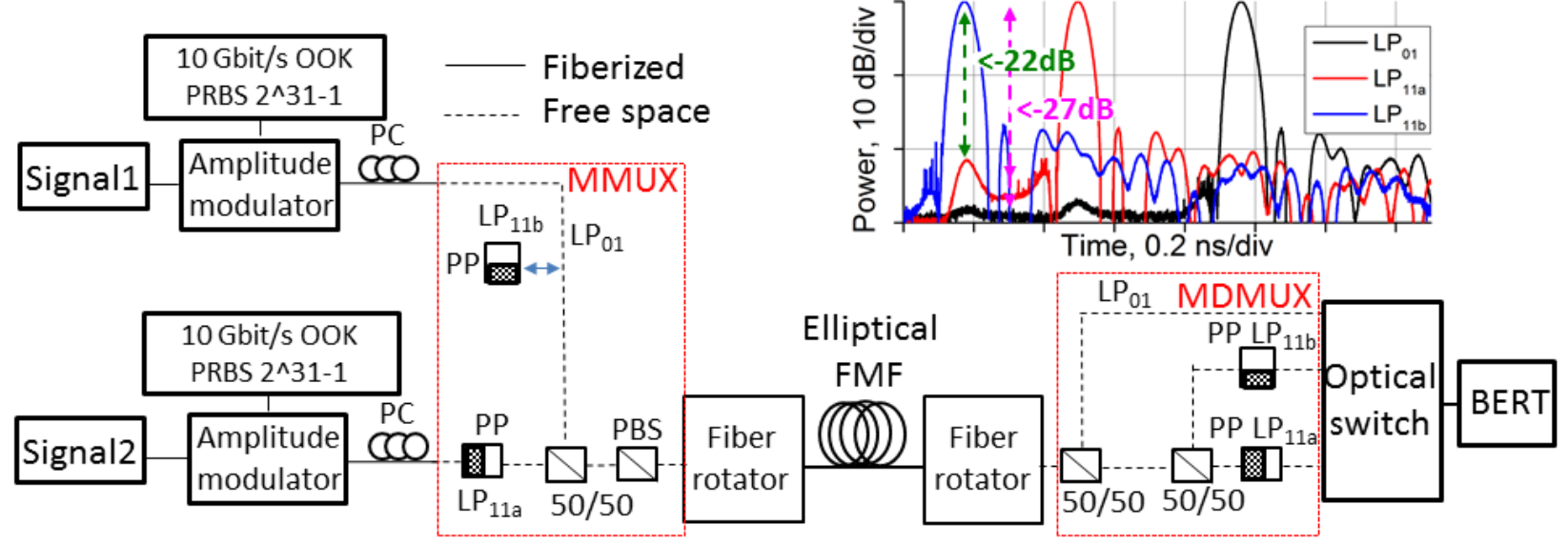

Fig. 1: Experimental set-up of the MIMO-less SDM transmission. Inset: time-of-flight measurements.

Figure 1 shows the experimental set-up used for the SDM transmission. Two continuous wave (CW) lasers both at $1550 \mathrm{~nm}$ are independently modulated to generate 10Gbit/s OOK signals using $2^{31}-1$ pseudo random bit sequences 

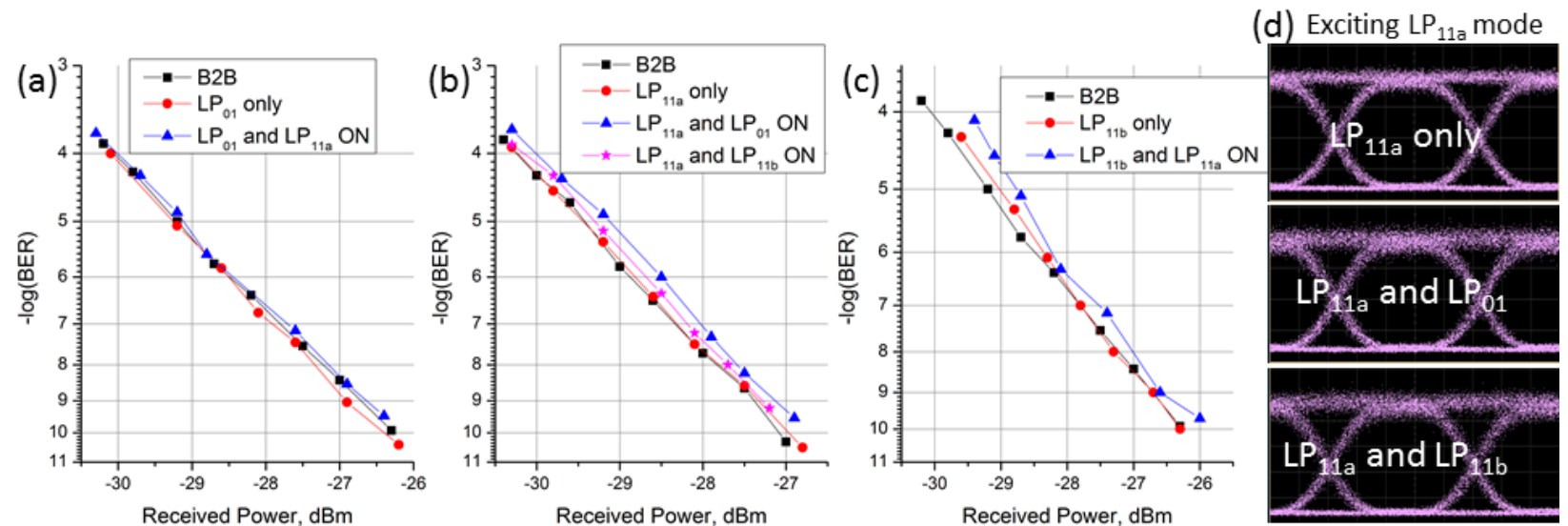

Fig. 2: BER curves for the various modes $\mathrm{LP}_{01}(\mathrm{a}), \mathrm{LP}_{11 \mathrm{a}}$ (b) and $\mathrm{LP}_{11 \mathrm{~b}}$ (c) with and without the other modes on, including back-to back curves. (d) Examples of eye diagrams of the signal transmitted in the $\mathrm{LP}_{11 \mathrm{a}}$ mode with and without the other signals transmitted in the $\mathrm{LP}_{01}$ and $\mathrm{LP}_{11 \mathrm{~b}}$ modes.

(PRBSs). One modulated laser beam is launched into the $\mathrm{LP}_{11 \mathrm{a}}$ mode, whereas a second is selectively launched into either the $\mathrm{LP}_{01}$ or the $\mathrm{LP}_{11 \mathrm{~b}}$ mode. In all cases, this was achieved by using a free-space mode multiplexer (MMUX), including phase plates (PPs) and a 50/50 combiner. Polarization controllers (PCs) and a polarization beam splitter (PBS) are used to ensure that the launched waves are co-polarized and aligned to the principal axis of the FMF. High precision fiber rotators were used at the input/output end of the FMF to align the angular position of the elliptic core of the FMF with the input $\mathrm{LP}_{11 \mathrm{a}} \mathrm{PP}$ and to minimize modal crosstalk. The power of each signal at the input of the FMF was about $-2 \mathrm{dBm}$. The FMF is a 1-km long graded-index fiber with a trench. The fiber supports $\mathrm{LP}_{01}$ and both $\mathrm{LP}_{11}$ modes, similar to the fiber described in [6]. However, unlike the fiber in [6,] this fiber has an elliptical core with an ellipticity of $\sim 10 \%$, which effectively guarantees a break in the spatial degeneracy of the $\mathrm{LP}_{11}$ mode, thus allowing minimal linear coupling between the $\mathrm{LP}_{11 \mathrm{a}}$ and $\mathrm{LP}_{1 \mathrm{~b}}$ modes, without breaking the polarization degeneracy of each mode [7]. The modal purity with which we are able to excite each spatial mode is mainly limited by our MMUX and is better than $22 \mathrm{~dB}$, while the distributed crosstalk among any of the spatial modes is better than $27 \mathrm{~dB}$, as time-of-flight measurements highlight, see inset to Fig.1. At the FMF output, we extract the different spatial modes using a free-space mode-demultiplexer (MDMUX), including again 50/50 splitters and PPs and launch the light into single-mode fibers. An optical switch followed by an optical pre-amplified receiver enables the selection and independent measurement of each MDMUX output in terms of eye diagrams and bit error ratio (BER) curves.

Figures 2 (a)-(c) show the BER curves as a function of the received optical power for all of the three spatial modes $\mathrm{LP}_{11}, \mathrm{LP}_{01}$ and $\mathrm{LP}_{11}$, respectively, with and without the other signals transmitted in the other modes being present. The square black symbols in each graph correspond to the back-to-back (B2B) BER curves, measured directly after the amplitude modulators, and are included for reference. When only one signal is launched into the FMF, no power penalty is observed regardless of the mode this signal travels in. Similar discussions hold when the signals are launched into any pair of the modes supported by the fiber, clearly highlighting high modal purity and extremely low distributed modal cross-talk. Importantly, no error floor is present even when the signals are simultaneously launched into the modes of the same mode group, i.e. $\mathrm{LP}_{11 \mathrm{a}}$ and $\mathrm{LP}_{11 \mathrm{~b}}$. Figure 2 (d) shows some typical eye diagrams of the signal launched into $\mathrm{LP}_{11 \mathrm{a}}$ mode when the other signals are either absent or present in the $\mathrm{LP}_{01}$ and $\mathrm{LP}_{11 \mathrm{~b}}$ modes. No degradation can be observed in the presence of the other SDM signals.

\section{Conclusions}

We have demonstrated a MIMO-less SDM transmission at $1550 \mathrm{~nm}$ over $1 \mathrm{~km}$ of an elliptical core few mode fiber supporting the $\mathrm{LP}_{01}, \mathrm{LP}_{11 \mathrm{a}}$ and $\mathrm{LP}_{11 \mathrm{~b}}$ modes. Negligible power penalties with no error floors are observed for any pair of excited modes thanks to the low level of modal crosstalk in the FMF, which was below $-22 \mathrm{~dB}$.

This work has been partially supported by UK Engineering and Physical Sciences Research Council (EPSRC) (EP/I01196X, The Photonics Hyperhighway); http://doi.org/10.5258/SOTON/403885.

\section{References}

[1] D. J. Richardson, et al., Nature Photonics 7, 354-362, 2013.

[2] E. Ip et al., Opt. Express 23(13), 17120-17126, 2015.

[3] G. Milione, et al., OFC W1F.2, 2016.

[4] G. Milione, et al., PD2-1, OECC/PS, 2016.

[5] L. Wang, et al., Optics Letters, vol. 40, no. 24, pp. 5846-5849, 2015.

[6] L. Grüner-Nielsen, et al., Journal of Lightwave Technology, vol. 30, no. 23, pp. 3693-3698, 2012.

[7] F. Parmigiani, et al., Th1F.4 OFC 2017. 